\title{
Main Neuralgias in Otolaryngology
}

\author{
Juan Antonio Lugo Machado* \\ Department of Otolaryngology, Sonora, México
}

*Corresponding author: Juan Antonio Lugo Machado, Department of Otolaryngology, Sonora, México

\section{Introduction}

The headache and neck are mediated by sensory fibers transmitted some cranial nerves, trigeminal, intermediary nerve, vagus and upper cervical spinal roots through the occipital and auricular nerves [1].

Nerve pain may have paroxysmal quality, which is usually of maximum onset and is often described as excruciating, similar to electric shock or puncture. There may be a single acute pain or repetitive aches in succession. The pain may last a fraction of a second or last several seconds. There may be a refractory period after severe pain during which pain will not occur. The mechanisms of neuropathic pain are complex. A nerve injury can induce peripheral and central changes that contribute to persistent pain and abnormal sensation. These processes end in normal circumstances as tissues heal and inflammation decreases [2]. Trigeminal neuralgia is one of the most common causes of facial pain. The pain tends to appear in the paroxysms and is maximum at the beginning. The pain has been described as an electric shock or puncture. Trigeminal painful neuropathy is defined by the head and/or facial pain in the distribution of one or more branches of the trigeminal nerve caused by another disorder and indicative of neural damage potential causes include acute herpes zoster, post herpetic neuralgia, and Trauma. Post-herpetic neuralgia is defined as the pain that persists between one and six months after the acute herpes zoster eruption has healed [1,2] painful posttraumatic trigeminal neuropathy, also known as painful anesthesia, is characterized by unilateral facial or oral pain that occurs after a traumatic trigeminal nerve injury, accompanied by additional symptoms or signs as a nerve dysfunction $[1,2]$. This condition is a perfect example of how difficult it can be to describe the central pain as the patient has a sensory loss, but at the same time may feel pain. In painful anesthesia, the pain overlaps in an area of the face that is lacking or has a sense of deterioration.

Diagnostic criteria for post-traumatic painful trigeminal neuropathy require all the following a) Facial and/or oral pain in the distribution of one or both trigeminal nerves

b) History of a traumatic event identifiable in the trigeminal nerve, with clinically evident positive signs (Hyperalgesia, Alodinia) and/or negative (Hypesthesia, hipoalgesia) of trigeminal nerve dysfunction.

Evidence of causality demonstrated by the following two:

a) Pain is found in the distribution of the trigeminal nerve affected by the traumatic event

b) The pain has developed within six months of the traumatic event.

c) It is not better explained by another diagnostic.

Trigeminal trophic syndrome, characterized by facial skin ulceration and Dysesthesia in relation to damage to the trigeminal nerve that affects any of the peripheral components, such as the trigeminal nerve (Including the trigeminal ganglion and sensory root) or its central sensory nuclei the most frequent causes are the therapeutic ablation of the trigeminal nerve and the medullary or Pontino ischemic stroke; Others include craniofacial surgery, trauma, and herpes zoster infection the most common symptoms of trigeminal trophic syndrome are dysesthesia annoying, such as itching, tingling, creeping, burning, as well as sensations of obstruction of the air flow Nasal and foreign ocular body. About half of the affected individuals report facial pain. The test reveals Hypesthesia or facial anesthesia. Skin ulceration tends to occur in the distribution of the infraorbital nerve, although it may occur in other trigeminal distros. There is No good treatment for Trigeminal trophic syndrome, although gabapentin and carbamazepine are often used in efforts to control neuropathic symptoms [3-9]. Cluster-Tic syndrome is a combination of cluster headache with coexistent trigeminal neuralgia characterized by three types of pain $[10-12]$. A component resembles trigeminal neuralgia pain and is 
paroxysmal, short-lived and severe. The second component is more like a cluster headache, albeit variable in length, with autonomic phenomena (e.g., ptosis, Miosis, tearing, conjunctival injection, rhinorrhea, nasal congestion). The third type of pain is a mixture of the first two and can be provoked by trigger points or by moving the neck. Cluster syndrome usually affects patients between 20 and 70 years of age. It may exist in chronic or episodic form (remissions and recurrences).

Glossopharyngealal neuralgia is defined as paroxysmal pain in areas innervated by the glossopharyngeal nerve and the auricular and pharyngeal branches of the vagus nerve there are numerous analogies with trigeminal neuralgia, with which it occasionally coexists, although the Glossopharyngeal neuralgia is much less common. Glossopharyngeal neuralgia is characterized by paroxysmal, severe, and stabbing pain that affects the ear, amygdalin fossa, base of the tongue, or below the angle of the mandible. Bilateral involvement has been reported in 12 percent of patient's typical triggers include chewing, swallowing, coughing, talking, yawning, certain tastes, or touching the neck or external auditory duct pain usually radiates towards Up from the oropharynx to the ear. The duration of severe paroxysms is from seconds to minutes, but there may also be a constant low-grade deaf background pain. Several dozen attacks can occur per day, sometimes waking the sleep patient. Some episodes may also be associated with an intense cough and/or hoarseness. Severe attacks have rarely been associated with bradycardia/asystole that cause syncope, probably because the entrance of the cranial nerve IX in the solitary tract has an effect on the dorsal motor nucleus of the cranial nerve X [11-17]

Diagnostic criteria for glossopharyngeal neuralgia, according to ICHD-3, require all of the following:

Recurrent paroxysmal attacks of unilateral pain in the distribution of the glossopharyngealic nerve

Pain has all four following characteristics:

i. Lasting from a few seconds to two minutes.

ii. Severe intensity.

iii. As electric shocks, gunshots, stabbings or acute quality.

iv. Precipitates when swallowing, coughing, talking or yawning.

v. Is not better explained by another diagnosis [1]

As with trigeminal neuralgia, there are idiopathic and secondary forms of glossopharyngeal neuralgia. Secondary causes include demyelinating lesions, cerebellopontine angle tumor, peritonsillar abscess, carotid aneurysm and Eagle syndrome (in which the cranial nerve IX is compressed laterally against an ossified ligament) [18, 19].The evaluation of a patient with suspected glossopharyngeal neuralgia includes a thorough history, especially on the presence of triggers. Careful intraoral and neck examination should be done to help exclude local disease as a cause of pain [20,21]. imaging studies are suitable for discarding calcifications of the estilofaringeo ligament (Eagle syndrome) medical treatment of glossopharyngeal neuralgia is essentially the same as for trigeminal neuralgia. In addition, the application of local anesthetics to the oropharynx can prove both diagnostic and therapeutic. Surgical treatment is considered for patients who fail in medical therapy.

The neuralgias of the intermediary nerve of the facial nerve, is a rare disorder characterized by short paroxysms of pain that is deeply felt in the auditory canal. Other terms used are geniculada neuralgia or hunting neuralgia. Occipital neuralgia may be a cause of headache in the occipital region. It is described as a stabbing pain paroxysmal in the distribution of the occipital nerve major, minor and third, sometimes accompanied by decreased sensitivity or dysesthesia in the affected area. There may be sensitivity to the affected nerve. The third occipital head ache is similar to greater occipital neuralgia, but much less common. [22-24].

\section{Conclusion}

Neuralgia is defined as paroxysmal pain in the distribution of a nerve, which otherwise has a normal function, while neuropathy is defined as an alteration of function or a pathological change in a nerve or nerves. Painful cranial neuralgias and neuropathies that because craniofacial pain include the following:

a) Trigeminal neuralgia, characterized by recurrent paroxysms of pain in the distribution of one or more branches of the cranial nerve $\mathrm{V}$.

b) Trigeminal neuropathy Painful, defined by head ache and/ or facial in the distribution of one or more branches of the trigeminal nerve caused by another disorder and indicative of neural damage. Glossopharyngealal Neuralgia, defined as paroxysmal pain in areas innervated by cranial nerves IX and X.

c) The neuralgia of the medial nerve, a rare disorder characterized by short paroxysms of pain that are felt in the auditory canal.

d) Occipital neuralgia, characterized by paroxysmal stabbing pain in the distribution of the occipital nerve greater, lower or Third, sometimes accompanied by decreased sensitivity or dysesthesia in the affected area.

\section{References}

1. Headache Classification Committee of the International Headache Society (IHS) (2018) The International Classification of Headache Disorders ( $3^{\text {rd }}$ edn), Cephalalgia 38(1): 1-211.

2. Rowbotham MC (2005) Mechanisms of neuropathic pain and their implications for the design of clinical trials. Neurology 65(12 Suppl 4): 66-73.

3. Elloumi Jellouli A, Ben Ammar S, Fenniche S, Zqhal M, Marrak H, et al. (2003) Trigeminal trophic syndrome: a report of two cases with review of literature. Dermatol Online J 9(5): 26.

4. Sadeghi P, Papay FA, Vidimos AT (2004) Trigeminal trophic syndrome-report of four cases and review of the literature. Dermatol Surg 30(5): 807-812. 
5. Garza I (2008) The trigeminal trophic syndrome: an unusual cause of face pain, dysaesthesias, anaesthesia and skin/soft tissue lesions. Cephalalgia 28(9): 980-985.

6. Sawada T, Asai J, Nomiyama T, Masuda K, Takenaka H, et al. (2014) Trigeminal trophic syndrome: report of a case and review of the published work. J Dermatol 41(6): 525-528.

7. Golden E, Robertson CE, Moossy JJ, Sandroni P, Garza I (2015) Trigeminal trophic syndrome: A rare cause of chronic facial pain and skin ulcers. Cephalalgia 35(7): 636

8. Collyer S, Fuller G (2012) Trigeminal trophic syndrome. Pract Neurol 12(5): 341.

9. Willis M, Shockley WW, Mobley SR (2011) Treatment options in trigeminal trophic syndrome: a multi-institutional case series. Laryngoscope 121(4): 712-716.

10. Watson P, Evans R (1985) Cluster-tic syndrome. Headache 25(3): 123 126.

11. Alberca R, Ochoa JJ (1994) Cluster tic syndrome. Neurology 44(6): 996

12. Mulleners WM, Verhagen WI (1996) Cluster-tic syndrome. Neurology 47(1): 302

13. Pascual J, Berciano J (1993) Relief of cluster-tic syndrome by the combination of lithium and carbamazepine. Cephalalgia 13(3): 205-206.

14. Solomon S, Apfelbaum RI, Guglielmo KM (1985) The cluster-tic syndrome and its surgical therapy. Cephalalgia 5(2): 83-89.

15. Rozen TD (2004) Trigeminal neuralgia and glossopharyngeal neuralgia.
Neurol Clin 22(1): 185-206

16. Rushton JG, Stevens JC, Miller RH (1981) Glossopharyngeal (vagoglossopharyngeal) neuralgia: a study of 217 cases. Arch Neurol 38(4): 201-205.

17. Elias J, Kuniyoshi R, Carloni WV, Borges MR, Peixoto CA, et al. (2002) Glossopharyngeal neuralgia associated with cardiac syncope. Arq Bras Cardiol 78(5): 510-519.

18. Bruyn GW (1983) Glossopharyngeal neuralgia. Cephalalgia 3(3):143157.

19. Kim E, Hansen K, Frizzi J (2008) Eagle syndrome: case report and review of the literature. Ear Nose Throat J 87(11): 631-633.

20. Soh KB (1999) The glossopharyngeal nerve, glossopharyngeal neuralgia and the Eagle's syndrome--current concepts and management. Singapore Med J 40(10): 659-665.

21. Patel A, Kassam A, Horowitz M, Chang YF (2002) Microvascular decompression in the management of glossopharyngeal neuralgia: analysis of 217 cases. Neurosurgery 50(4): 705-710.

22. Franzini A, Messina G, Franzini A (2017) Treatments of glossopharyngeal neuralgia: towards standard procedures. Neurol Sci 38 (Suppl 1): 51-55.

23. Spina A, Boari N, Gagliardi F, Bailo M, Morselli C, et al. (2017) The emerging role of gamma knife radiosurgery in the management of glossopharyngeal neuralgia. Neurosurg Rev.

24. Borius PY, Tuleasca C, Muraciole X, Negretti L, Reqis J, et al. (2018) Gamma Knife radiosurgery for glossopharyngeal neuralgia: A study of 21 patients with long-term follow-up. Cephalalgia 38(3): 543-550.

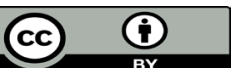

This work is licensed under Creative Commons Attribution 4.0 License

To Submit Your Article Click Here:

Submit Article
DOI: $10.32474 /$ SJO.2019.01.000117

Citation: Juan Antonio Lugo M. Main Neuralgias in Otolaryngology. Sch J Oto 1(4)-2018. SJO. MS.ID.000117. D0I: 10.32474/

SJ0.2019.01.000117.

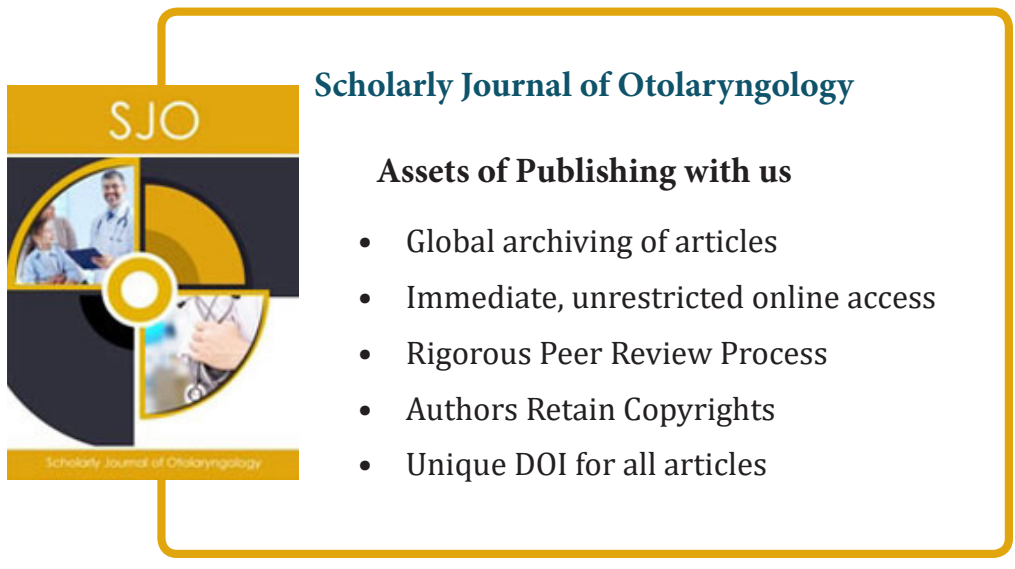

